\title{
Carotid intima-media thickness and arterial stiffness as early markers of atherosclerosis in pediatric celiac disease
}

\author{
Arzu Meltem Demir ${ }^{1}$ Zarife Kuloğlu¹, Aytaç Yaman, Suat Fitöz², Gökhan Nergizoğlu³, \\ Aydan Kansu ${ }^{1}$ \\ Division of ${ }^{1}$ Pediatric Gastroenterology, Department of Pediatrics, ${ }^{2}$ Department of Radiology and ${ }^{3}$ Department of Nephrology, \\ Ankara University Faculty of Medicine, Ankara, Turkey. \\ E-mail: arzuyusufoglu@yahoo.com \\ Received: 25 February 2016, Revised: 13 April 2016, Accepted: 31 May 2016
}

SUMMARY: Demir AM, Kuloğlu Z, Yaman A, Fitöz S, Nergizoğlu G, Kansu A. Carotid intima-media thickness and arterial stiffness as early markers of atherosclerosis in pediatric celiac disease. Turk J Pediatr 2016; 58: 172-179.

The association between pediatric celiac disease (CD) and atherosclerosis is unknown. Our aim was to investigate whether pediatric CD patients have an increased risk of atherosclerosis. We evaluated the premature atherosclerosis by pulse wave velocity (PWV) and carotid intima-media thickness (cIMT). A total of $37 \mathrm{CD}$ patients (20 girls, mean age $13 \pm 3.3$ years) and 36 healthy age and sex matched controls were enrolled. Mean duration of CD was 47.1 \pm 32.3 months and $40.5 \%$ of patients had positive tissue transglutaminase antibody (tTg) IgA. Total cholesterol level was lower in CD $(p=0.026)$ and cIMT was lower in tTg IgA antibody negative CD $(\mathrm{p}=0.030)$. cIMT was significantly correlated with tTg IgA antibody positivity $(r=0.336 ; p=0.042)$. Adherence to strict gluten-free diet is associated with decreased cIMT, suggesting that gluten withdrawal seems to have a beneficial effect on premature atherosclerosis.

Key words: celiac disease, children, atherosclerosis, carotid intima media thickness, pulse wave velocity.

Celiac disease $(\mathrm{CD})$ is characterised by chronic immune-mediated small bowel inflammation after ingestion of dietary gluten in genetically predisposed individuals ${ }^{1}$. Recently, increased cardiovascular disease (CVD) risk due to chronic inflammation has been described in adult celiac patients, although limited data is available ${ }^{2,3}$.

Early diagnosis of preclinical atherosclerosis can be made by measurement of carotid artery intima-media thickness (cIMT), flow-mediated vasodilatation and arterial stiffness ${ }^{4}$. Carotid artery intima-media thickness is a reliable surrogate marker of atherosclerosis ${ }^{5,6}$. Pulse wave velocity (PWV) is the main index for estimating arterial elasticity. This reliable, repeatable and noninvasive method is easy to practice and useful in detecting increased stiffness at the early stage ${ }^{7}$. Pulse wave velocity can be measured by applanation tonometry, oscillometry or vascular ultrasound on several arterial sites ${ }^{8}$. The parameter carotid-femoral
PWV mainly reflects stiffness of the artery ${ }^{9}$. PWV and augmentation index are independent predictors of cardiovascular mortality in adults $^{10}$.

Limited studies in adult celiac patients showed that preclinical atherosclerosis might be prevalent. However, there is no data about preclinical atherosclerosis in pediatric CD. Atherosclerotic process begins in childhood which progresses slowly without ischemic complications such as myocardial infarction or stroke ${ }^{11}$. Therefore, modification of the disease in this crucial period may have the greatest impact. The arterial thickness and distensibility, endothelial function, and the proinflammatory and prothrombotic state, alone or in combination can be used as a method for identifying children who have the highest risk for CVD in adulthood, and to form specific treatment strategies. All of these findings led us to perform the present study to investigate the arterial thickness and distensibility by 
measuring cIMT and PWV as earlier signs of atherosclerotic vascular changes in children with CD as compared with healthy controls.

\section{Material and Methods}

This prospective, observational, cross-sectional, single center study was performed in the Pediatric Gastroenterology Department, Ankara University School of Medicine, between January 2013 and March 2014. Study population included individuals with previously diagnosed $\mathrm{CD}$ and age and sex matched healthy controls. Inclusion criteria for celiac patients included the following: male or female patient older than the age 6 years and younger than 18 years with biopsy-proven $\mathrm{CD}$ according to European Society for Pediatric Gastroenterology, Hepatolgy and Nutrition (ESPGHAN) criteria and not having the exclusion criteria. Information on disease duration, clinical type and histopathological type according to modified Marsh-Oberhuber classification ${ }^{12}$ were recorded in patients with CD. Healthy control subjects were defined as without known chronical illness at the time of enrollment and not having the exclusion criteria. The following exclusion criteria was used for both patients and controls: Age more than 18 or less than 6 years at entry, comorbid disease/condition such as diabetes mellitus, obesity, hypertension, dyslipidemia, systemic autoimmune disease, active infection, evidence of liver, renal and lung disease, smoking exposure, concurrent treatment with antihypertensive drugs, lipid-lowering drugs and positive family history for dyslipidemia and early coronary arterial disease.

A clinical examination including weight, height and blood pressure measurement was performed in all subjects by standard methods and by the same pediatrician. Body mass index (BMI) was calculated using standard weight $(\mathrm{kg}) /$ height $(\mathrm{m})^{2}$ formula and values were evaluated with percentile cards prepared for Turkey according to gender and age. Values above the 95th percentile were accepted as indicating obesity ${ }^{13}$.

Systolic and diastolic blood pressures (BP) were measured in the sitting position by a mercury sphygmomanometer three times, and the means were calculated. After overnight fasting for at least 12 hours, a venous peripheral blood sample was obtained for lipid profile [triglycerides, total cholesterol, high density lipoprotein (HDL), low density lipoprotein (LDL), and very low density lipoprotein (VLDL)], C reactive protein (CRP), erytrocyte sedimentation rate (ESR), $25(\mathrm{OH})$ vitamin $\mathrm{D}$, serum immunglobulin (Ig) A, tissue transglutaminase (tTg) IgA antibody and $\mathrm{HbAlc}$ in each participant on the same day of clinical examination, tonometry and cIMT evaluation. The samples were analyzed in local laboratory. IgA-class tTg antibody was tested by ELISA using a commercial kit (Organtec Diagnostics, Mainz, Germany) and a value higher than 10 $\mathrm{U} / \mathrm{mL}$ was considered positive. Serum HbAlc and $25(\mathrm{OH})$ vitamin D levels were measured by high-performance liquid chromatography (HPLC, Shimadzu, Kyoto, Japan). Lipid profile, plasma glucose and CRP levels were analyzed with Siemens Advia 1800 chemistry system (Siemens Healthcare Diagnostics, Tarrytown NY), ESR was measured by Westergen method.

\section{Carotid intima-media thickness}

Carotid artery intima-media thickness was measured by a single experienced pediatric radiologist who was unaware of clinical and laboratory characteristics of the children. The common carotid arteries were evaluated in anterolateral, posterolateral and medial directions with a central frequency $12-\mathrm{MHz}$ linear array transducer and an Aplio scanner (Toshiba Medical Systems Co, Ltd, Tokyo, Japan). Measurements were performed $1 \mathrm{~cm}$ proximal to the carotid bulb. Longitudinal images of the carotid arteries were obtained in the supine position with head turned 45 degree to contralateral side. The intima-media thickness (IMT) was measured by tracing the distance from the leading-edge of the lumen intima to the media-adventitia interfaces. Three examinations were taken from both common carotid arteries and means were calculated to estimate the overall IMT. cIMT was assessed by sex-specific curves for age and increased cIMT was defined as cIMT $>90 \%$, which shows atherosclerosis ${ }^{14}$.

\section{Assessment of endothelial function}

Pulse wave velocity was measured by applanation tonometry using a SphygmoCor Version 7.0 Vx (AtCor Medical, Sydney, Australia) device in a supine position after resting 10 minutes. The probe was connected to a handheld electrocardiograph (ECG) unit while pressure and electrocardiographic signals were 
transmitted to a computer (Exper, Datateknik, Istanbul, Turkey). Aortic PWV was measured in sequential recordings of carotid and femoral arterial pressure waves and defined as the distance between sampling sites divided by the time delay between the distal and proximal pulses according to the $\mathrm{R}$ wave belonging to the ECG QRS complex and calculated by software. Two pulse wave passes were examined: (i) between the carotid site and the jugular notch, and (ii) between the jugular notch and the femoral site. The difference between these distances was recorded as the distance covered by the pulse wave. Later, three ECG electrodes connected to the device were placed on the patient's body, and carotid-femoral PWV was recorded. Each measurement was done three times and means calculated. PWV was assessed by sex-specific curves for age and height, and abnormal PWV was defined as PWV $>90 \%$, which shows arterial stiffness ${ }^{15}$.

\section{Statistical analysis}

Statistical analysis was done using SPSS version 11.5. Continuous variables were presented as mean \pm SD or median, and categorical variables were presented as frequency (percentage). The chi-square test was used to evaluate differences for categorical variables. For variables with normal distributions, $T$ test for two groups was used. For the variables failing the normality test, statistical significance between groups was determined by non-parametric MannWhitney $U$ test. An assessment of the relation between PWV or cIMT and specific variables including age, height, weight, BMI, systolic/ diastolic blood pressure, HbA1c, lipid profile, sedimentation, CRP, $25(\mathrm{OH})$ vitamin $\mathrm{D}$ and tTg IgA was performed using Spearman/Pearson

Table I. Demographic, Anthropometric and Biochemical Data of the Study Groups

\begin{tabular}{|c|c|c|c|}
\hline & $\begin{array}{c}\mathrm{CD} \\
(\mathrm{n}=37)\end{array}$ & $\begin{array}{l}\text { Healthy Controls } \\
\qquad(\mathrm{n}=36)\end{array}$ & $\mathrm{P}$ \\
\hline Age (years)* & $13 \pm 3.3$ & $12.7 \pm 3.6$ & 0.630 \\
\hline Sex (M) (\%) & 45.9 & 44.4 & 0.897 \\
\hline Weight $(\mathrm{kg})^{*}$ & $39.6 \pm 14.1$ & $43.3 \pm 16$ & 0.302 \\
\hline Height $(\mathrm{cm})^{*}$ & $146.9 \pm 15.9$ & $151.1 \pm 19$ & 0.305 \\
\hline BMI $\left(\mathrm{kg} / \mathrm{m}^{2}\right)^{*}$ & $17.6 \pm 2.84$ & $18.1 \pm 3.1$ & 0.433 \\
\hline Systolic BP (mm Hg)\# & $100(90-170)$ & $102.5(90-140)$ & 0.349 \\
\hline Diastolic BP (mm Hg)\# & $65(50-110)$ & $70(60-90)$ & 0.295 \\
\hline HbAlc (\%)\# & $5.1(3.9-5.7)$ & $5.1(4-5.4)$ & 0.294 \\
\hline Triglycerides (mg/dl)\# & $61(25-147)$ & $61(10-185)$ & 0.667 \\
\hline Total cholesterol (mg/dl)\# & $142(24-206)$ & $155(102-222)$ & 0.026 \\
\hline HDL cholesterol (mg/dl)\# & $45(28.3-86.5)$ & $51.5(36-73)$ & 0.081 \\
\hline LDL cholesterol (mg/dl)\# & $84(29-148)$ & $87.5(43-154)$ & 0.138 \\
\hline VLDL cholesterol (mg/dl)\# & $12(5-29)$ & $12(2-37)$ & 0.596 \\
\hline $25(\mathrm{OH})$ vitamin $\mathrm{D}(\mu \mathrm{g} / \mathrm{L}) \#$ & $26.6(10-59.1)$ & $20.2(5.4-49.1)$ & 0.089 \\
\hline Sedimentation $(\mathrm{mm} / \mathrm{h}) \#$ & $10(2-30)$ & $9(1-44)$ & 0.956 \\
\hline CRP (mg/L)\# & $1(1-8.7)$ & $1(0-8.2)$ & 0.730 \\
\hline tTg IgA antibody $(\mathrm{U} / \mathrm{L}) \#$ & $3.8(0.3-395.7)$ & $0.3(0.1-1.3)$ & 0,000 \\
\hline $\operatorname{PWV}(\mathrm{m} / \mathrm{s})^{*}$ & $4.9 \pm 0.8$ & $4.7 \pm 0.6$ & 0.646 \\
\hline Abnormal PWV, n (\%) & $16(43.2)$ & $9(25)$ & 0.101 \\
\hline cIMT $(\mathrm{mm})^{*}$ & $0.41 \pm 0.06$ & $0.42 \pm 0.04$ & 0.111 \\
\hline
\end{tabular}

Data are *mean \pm SD or \#median (min-max)

CD: celiac disease; BMI: body mass index; BP: blood pressure; HbAlc: hemoglobin A1c; HDL: high density lipoprotein; LDL: low density lipoprotein; VLDL: very low density lipoprotein; CRP: C reactive protein; tTg IgA: tissue transglutaminase IgA; PWV: pulse wave velocity, cIMT: carotid intima-media thickness 
Table II. Comparative Analysis of Vascular Parameters Between Age and Sex Matched Controls and tTg IgA Positive and Negative Celiac Patients

\begin{tabular}{lcccccc}
\hline & $\begin{array}{c}\text { tTg IgA } \\
\text { Positive } \\
\text { CD } \\
(\mathrm{n}=15)\end{array}$ & $\begin{array}{c}\text { Healthy } \\
\text { Controls } \\
(\mathrm{n}=36)\end{array}$ & $\mathrm{p}$ & $\begin{array}{c}\text { tTg IgA } \\
\text { Negative } \\
\text { CD }\end{array}$ & $\begin{array}{c}\text { Healthy } \\
\text { Controls } \\
(\mathrm{n}=36)\end{array}$ & $\mathrm{p}$ \\
\hline PWV $(\mathrm{m} / \mathrm{s}){ }^{*}$ & $4.9 \pm 0.9$ & $4.7 \pm 0.6$ & 0.876 & $4.9 \pm 0.7$ & $4.7 \pm 0.6$ & 0.431 \\
Abnormal PWV, n (\%) & $6(40)$ & $9(25)$ & 0.325 & $10(45)$ & $9(25)$ & 0.107 \\
cIMT $(\mathrm{mm}){ }^{*}$ & $0.43 \pm 0.08$ & $0.42 \pm 0.04$ & 0.557 & $0.4 \pm 0.05$ & $0.42 \pm 0.04$ & 0.03 \\
\hline
\end{tabular}

Data are *mean \pm SD

CD: celiac disease; tTg IgA: tissue transglutaminase IgA; PWV: pulse wave velocity; cIMT: carotid intima-media thickness

Rank correlation test. All statistical significance was defined as $\mathrm{p}<0.05$ (2-tailed).

\section{Ethical consideration}

This study was approved by the ethics committee. A written informed consent was given and signed by parents or caregivers and from the patients aged $>10$ years after detailed information on the procedures. The study was conducted in accordance with the Declaration of Helsinki guidelines.

\section{Results}

A total of 80 patients (40 celiac patients and 40 controls), aged 6-18 years, were screened and provided baseline measurements. Of these patients, 7 subjects ( 3 celiac patients and 4 controls) were withdrawn from the statistical analysis. Five subjects ( 1 celiac patients and 4 controls) had high $\mathrm{HbAlc}$ level $(>6 \%)$, while 2 celiac patients were on a gluten-free diet less than 12 months. The study enrolled a total of 37 celiac patients ( $54 \%$ female, mean age $13 \pm 3.3$ years) and 36 age and sex matched healthy children. Mean CD duration was $47.1 \pm 32.3$ months and 22 patients had classical, 11 atypical and 4 silent type $C D$, respectively. Histopathological analysis was compatible with Marsh Type II CD in 6, Marsh Type IIIa CD in 2, Marsh Type IIIb CD in 3 and Marsh Type IIIc $\mathrm{CD}$ in 26 children, respectively. Anti tTg IgA was positive in 15 children with CD $(40.6 \%)$. Six of them had classical (diarrhea, abdominal pain), 7 had atypical (short stature, anemia) and 2 had silent type CD. Histopathological analysis was Marsh Type IIIa CD in 2, Marsh Type IIIb in 1 and Marsh Type IIIc in 12 of Anti tTg IgA positive children. All of the Anti tTg IgA positive patients were not strictly adherent to gluten free diet (GFD). Based on the $\mathrm{tTg} \operatorname{IgA}$ at the enrollment, 22 (59.4\%) celiac patients were considered to be strictly adherent to gliadin free diet (GFD).

There was no difference between serum cholesterol levels in tTg IgA positive and negative $C D(p=0.433)$, but children with $C D$ had significantly lower serum cholesterol levels compared with healthy controls $(p=0.026)$. The remaining clinical and biochemical findings did not differ between the two groups (Table I). There was no significant difference between the tTg IgA positive and $\mathrm{tTg} \operatorname{IgA}$ negative celiac patients, in terms of PWV, abnormal PWV and $\operatorname{cIMT}(\mathrm{p}=0.795, \mathrm{p}=0.742, \mathrm{p}=0.262$, respectively). Celiac patients with tTg IgA negative had significantly lower cIMT than controls $(p=0.03)$ (Table II). Four $(26.7 \%)$ of tTg IgA positive patients had increased cIMT whereas only $1(4.5 \%)$ of $\mathrm{tTg}$ IgA negative patients had increased cIMT. There was no significant difference between two groups for cIMT $(p=0.136)$. In patients with tTg IgA positive and increased cIMT, the mean age of disease onset was $10.2 \pm 2.5$ (7-13.2) years, mean age of diagnosis was $11.8 \pm 2(9-13.5)$ years and mean time interval between the onset of disease and diagnosis was $18.7 \pm 14.3$ months (3-36 months). In patients with tTg IgA positive and normal cIMT, the mean age of disease onset was $8.5 \pm 4.5(2.9-16.10)$ years, the mean age of diagnosis was $10.8 \pm 3.9$ (4.6-17.10) years and mean time interval between the onset of disease and diagnosis was $27.5 \pm 41.4$ months (0-120 months). There was no significant difference for the time interval between the onset of disease and diagnosis in tTg IgA positive patients with increased cIMT or normal cIMT $(p=0.661)$. The clinical and biochemical findings of $\mathrm{tTg}$ IgA positive and negative patients were shown in Table III. 
Table III. Comparative Analysis of Demographic, Anthropometric and Biochemical Data of the Tissue Transglutaminase Positive and Negative Patients

\begin{tabular}{lccc}
\hline & $\begin{array}{c}\text { CD with tTg IgA(+) } \\
(\mathrm{n}=15)\end{array}$ & $\begin{array}{c}\text { CD with tTg IgA }(-) \\
(\mathrm{n}=22)\end{array}$ & $\mathrm{P}$ \\
\hline Age (years)* & $14.5 \pm 3$ & $12 \pm 3.22$ & 0.024 \\
Sex (M) (\%) & 53.3 & 40.9 & 0.457 \\
CD duration (months)* & $43.2 \pm 36$ & $47.7 \pm 32.7$ & 0.680 \\
BMI (kg/m²)* & $18.6 \pm 2.5$ & $16.9 \pm 2.9$ & 0.067 \\
Systolic BP (mm Hg)\# & $105(90-170)$ & $100(90-140)$ & 0.576 \\
Diastolic BP (mm Hg)\# & $65(50-110)$ & $65(60-90)$ & 0.548 \\
HbAlc (\%)\# & $5(3.9-5.6)$ & $5(4.3-5.7)$ & 0.898 \\
Triglycerides (mg/dl)\# & $68(34-147)$ & $50(25-141)$ & 0.319 \\
Total cholesterol (mg/dl)\# & $133(99-206)$ & $147(95-205)$ & 0.433 \\
HDL cholesterol (mg/dl)\# & $43(29-86.5)$ & $49.1(28-71.5)$ & 0.496 \\
LDL cholesterol (mg/dl)\# & $72.3(55.2-148)$ & $85(29-128)$ & 0.746 \\
VLDL cholesterol (mg/dl)\# & $14(7-29)$ & $10.5(5-28)$ & 0.334 \\
25 (OH) vitamin D (ug/L)\# & $21.4(16-54.6)$ & $28.1(16.5-59.1)$ & 0.056 \\
Sedimentation (mm/h)\# & $10(2-30)$ & $10(2-22)$ & 0.400 \\
CRP (mg/L)\# & $1(1-1.3)$ & $1(1-8.7)$ & 0.891 \\
tTg IgA antibody (U/L)\# & $83.8(13-395.7)$ & $1.5(0.3-11.2)$ & 0.000 \\
PWV (m/s)* & $4.9 \pm 0.9$ & $4.9 \pm 0.7$ & 0.795 \\
Abnormal PWV n, (\%) & $6(40)$ & $10(45.5)$ & 0.742 \\
cIMT (mm)* & $0.43 \pm 0.08$ & $0.4 \pm 0.05$ & 0.262 \\
\hline
\end{tabular}

Data are *mean \pm SD or \#median (min-max)

CD: celiac disease; BMI: body mass index; BP: blood pressure; HbAlc: hemoglobin A1c; HDL: high density lipoprotein; LDL: low density lipoprotein; VLDL: very low density lipoprotein; CRP: C reactive protein; tTg IgA: tissue transglutaminase IgA; PWV: pulse wave velocity, cIMT: carotid intima-media thickness

An 11 year-old male with CD had abnormal PWV, increased cIMT and atherosclerotic plaques in both carotid arteries. His clinical symptoms started at the age of 7 years. $\mathrm{He}$ had short stature and growth failure. He was diagnosed as CD with tTg IgA $>300 \mathrm{U} / \mathrm{ml}$ (Normal; 0-10) and histopathological analysis of Marsh type IIIc at the age of 9 years. He was not strictly adherent to the gluten free diet and his tTg IgA did not decrease to normal levels. He did not carry any other risk factor for atherosclerosis. Blood pressure, lipid profile, 25 $(\mathrm{OH})$ vitamin $\mathrm{D}$, sedimentation, and CRP were all normal. Renal artery Doppler USG, renin and aldosteron levels were in normal limits.

Celiac patients had a significant correlation between PWV and age $(r=0.360, p=0.029)$, weight $(r=0.384, p=0.019)$, height $(r=0.333$, $p=0.044)$, body mass index $(r=0.324, p=0.05)$, and systolic and diastolic blood pressure $(\mathrm{r}=0.454, \mathrm{p}=0.005$ and $\mathrm{r}=0.390, \mathrm{p}=0.017$ respectively). There was a significant positive correlation between cIMT and $\mathrm{tTg}$ IgA antibody level in children with $C D(r=0.336, p=0.042)$.

\section{Discussion}

This cross-sectional study is the first report to investigate the preclinical atherosclerosis by arterial stiffness and thickness using PWV and cIMT in pediatric celiac disease. We have demonstrated that after mean $47.1 \pm 32.3$ months of CD diagnosis, arterial stiffness and thickness are not different in CD compared with controls. The cIMT is positively correlated with tTg IgA antibody level in patients with $C D$, suggesting that uncontrolled or undiagnosed CD might influence arterial thickness. The time interval between clinical symptoms and diagnosis was not predictive due to the small sample size. In addition, we demonstrated that 
serum total cholesterol level is significantly lower in children with CD compared to controls.

There is limited and conflicting data published on cardiovascular complications in patients with $\mathrm{CD}^{2,3}$. A significant increment in mortality from CVD in patients with CD have been reported in some studies ${ }^{2,3}$, but not in the others ${ }^{16,17}$. The cardiovascular risk factors have not been investigated in children with CD until recently. To our knowledge, only one multicenter study explored 114 children with CD on GFD for at least one year. They showed $14 \%$ of children had one or more concomitant traditional CVD risk factors such as dyslipidemia or hypertension. They suggested screening for CVD risk factors in $\mathrm{CD}$ both at diagnosis and during follow-up is important ${ }^{18}$.

Inflammation plays a key role in the pathogenesis of atherosclerosis. Although a link between CD and CVD remains weak, it is considered that chronic inflammatory condition in $\mathrm{CD}$ may be directly responsible for the development of endothelial dysfunction, leading to a subsequent increase in arterial stiffness and atherosclerotic lesions. A few studies in adults have suggested that $\mathrm{CD}$ is associated with premature atherosclerosis ${ }^{19-21}$. Endothelial dysfunction at the macrovascular level was found in adult celiac patients ${ }^{21}$. Significant increase of cIMT and decreased endotheliumdependent dilatation were found in the young adults with $\mathrm{CD}$ compared to the controls; suggesting that they may have accelerated atherosclerosis. Adult celiac patients were also shown to have greater cIMT as compared to healthy individuals. Increased arterial stiffness was found in adult celiac patients compared to the controls.

All of these findings suggest that preclinical atherosclerosis might be prevalent in adult celiac patients. It is well known that atherosclerosis begins in childhood; however, children rarely experience cardiovascular events. Atherosclerosis may eventually lead to myocardial infarction or stroke during adulthood $^{11}$. However, there is no data about the pediatric population. Therefore, we investigated preclinical atherosclerosis in pediatric celiac patients by PWV and cIMT. There was no difference in arterial stiffness and thickness between patients with $\mathrm{CD}$ and controls. Young age of onset and longer disease duration with long-term gluten withdrawal in our study might prevent premature atherosclerosis. Interestingly, one of our patients had atherosclerotic plaques in both carotid arteries. He was supposed to be on GFD for 22 months; however his tTg IgA was positive. We have not found any other risk factor for atherosclerosis such as renal or endocrin disorders. Uncontrolled CD may be the probable cause of atherosclerosis in this child. Development of atherosclerosis may be related to tTg IgA positivity, reflecting inflammatory activity.

The influence of GFD on the risk factors of CVD remains unclear. Some studies have suggested that GFD may have an anti-atherosclerotic effect in $\mathrm{CD}^{22-24}$, whereas others have shown that the diet is likely to be atherogenic ${ }^{25-27}$. Serum total cholesterol level was significantly lower in CD compared to healthy controls in our study as reported previously in the adults ${ }^{22,26}$. Lower cIMT in CD with strict gluten adherence suggest that GFD seems to have a beneficial effect on cardiovascular profile.

There is a well known relationship between the presence of celiac disease specific antibodies and the severity and prognosis of the disease ${ }^{28}$. In our study, we evaluated the association of celiac specific antibodies with PWV and cIMT. We found no significant difference between antibody positive and antibody negative patients in terms of PWV and CIMT. Similar results were also observed in two different studies ${ }^{20,21}$.

Interestingly, we found cIMT was significantly correlated with tTg IgA antibody level which is the marker of uncontrolled $\mathrm{CD}$, whereas traditional risk factors for atherosclerosis (ie body mass index, blood pressure) were not correlated. Indeed, we showed that arterial thickness was significantly lower in children with CD who were strictly adherent to GFD compared to healthy control. Our results suggest that the GFD is likely to be antiatherogenic. Similarly, in the study of De Marchi et al. ${ }^{19}$, adult patients with CD at baseline had significantly increased cIMT compared to the controls. After GFD, a significant decrease in cIMT was shown, they explained this difference by decrement of inflammatory response due to normalization of the small bowel mucosa secondary to gluten abstinence. And, signs of early atherosclerosis also improved rapidly with GFD in those patients. In accordance with this, 
children with uncontrolled celiac disease are at risk to develop early atherosclerosis; and premature atherosclerosis can be modified by strict GFD.

Increased arterial stiffness is correlated with the aging process and extrinsic risk factors of atherosclerosis such as hypertension, diabetes, dyslipidemia, obesity and smoking 9,10 . We found a positive correlation between PWV, body mass index and BP (both systolic and diastolic) in celiac patients as previously shown in healthy children ${ }^{13}$.

In conclusion, we demonstrated that $\mathrm{CD}$ is associated with low cholesterol levels and cIMT is significantly correlated with tTg IgA antibody level. Indeed, compared to healthy controls, celiac patients who are strictly adherent to GFD have significantly lower cIMT. These results suggest that gluten withdrawal seems to have a beneficial effect on atherosclerotic risk factors.

\section{Acknowledgement}

This study was funded by the National Pediatric Society.

\section{REFERENCES}

1. Kelly CP, Bai JC, Liu E, Leffler DA. Advances in diagnosis and management of coeliac disease. Gastroenterology 2015; 148: 1175-1186.

2. Viljamaa M, Kaukinen K, Pukkala E, Hervonen K, Reunala T, Collin P. Malignancies and mortality in patients with coeliac disease and dermatitis herpetiformis: 30-year population-based study. Dig Liver Dis 2006; 38: 374-380.

3. Wei L, Spiers E, Reynolds N, Walsh S, Fahey T, MacDonald TM. The association between coeliac disease and cardiovascular disease. Aliment Pharmacol Ther 2008; 27: 514-519.

4. McNeal CJ, Wilson DP, Christou D, et al. The use of surrogate vascular markers in youth at risk for premature cardiovascular disease. J Pediatr Endocrinol Metab 2009; 22: 195-211.

5. Lamotte C, Iliescu C, Libersa C, Gottrand F. Increased intima-media thickness of the carotid artery in childhood: a systematic review of observational studies. Eur J Pediatr 2011; 170: 719-729.

6. van den Oord SC, Sijbrands EJ, ten Kate GL, et al. Carotid intima-media thickness for cardiovascular risk assessment: systematic review and meta-analysis. Atherosclerosis 2013; 228: 1-11.

7. Bruno RM, Bianchini E, Faita F, Taddei S, Ghiadoni L. Intima media thickness, pulse wave velocity, and flow mediated dilation. Cardiovasc Ultrasound 2014; 12: 34 .
8. Ridha M, Nourse SE, Selamet Tierney ES. Paediatric interventions using noninvasive vascular health indices. Hypertension 2015; 65: 949-955.

9. Vlachopoulos C, Aznaouridis K, Stefanadis C. Prediction of cardiovascular events and all-cause mortality with arterial stiffness: a systematic review and meta-analysis. J Am Coll Cardiol 2010; 55: 1318-1327.

10. Mitchell GF, Hwang SJ, Vasan RS, et al. Arterial stiffness and cardiovascular events: the Framingham Heart Study. Circulation 2010; 121: 505-511.

11. Raitakari OT, Juonala $M$, Kähönen $M$, et al. Cardiovascular risk factors in childhood and carotid artery intima-media thickness in adulthood: the Cardiovascular Risk in Young Finns Study. JAMA 2003; 290: 2277-2283.

12. Corazza GR, Villanacci V. Coeliac disease. J Clin Pathol 2005; 58: 573-574.

13. Bundak R, Furman A, Gunoz H, Darendeliler F, Bas F, Neyzi O. Body mass index references for Turkish children. Acta Paediatr 2006; 95: 194-198.

14. Curcio S, García-Espinosa V, Arana M, et al. GrowingRelated Changes in Arterial Properties of Healthy Children, Adolescents, and Young Adults Nonexposed to Cardiovascular Risk Factors: Analysis of GenderRelated Differences. Int J Hypertens 2016; 2016: 4982676.

15. Reusz GS, Cseprekal O, Temmar M, et al. Reference values of pulse wave velocity in healthy children and teenagers. Hypertension 2010; 56: 217-224.

16. West J, Logan RF, Card TR, Smith C, Hubbard R. Risk of vascular disease in adults with diagnosed coeliac disease: a population-based study. Aliment Pharmacol Ther 2004; 20: 73-79.

17. Whorwell PJ, Alderson MR, Foster KJ, Wright R. Death from ischaemic heart-disease and malignancy in adult patients with coeliac disease. Lancet 1976; 2: $113-114$

18. Norsa L, Shamir R, Zevit N, et al. Cardiovascular disease risk factor profiles in children with coeliac disease on gluten-free diets. World J Gastroenterol 2013; 19: 5658-5664

19. De Marchi S, Chiarioni G, Prior M, Arosio E. Young adults with coeliac disease may be at increased risk of early atherosclerosis. Aliment Pharmacol Ther 2013; 38: $162-169$.

20. Korkmaz H, Sozen M, Kebapcilar L. Increased arterial stiffness and its relationship with inflammation, insulin, and insulin resistance in coeliac disease. Eur J Gastroenterol Hepatol 2015; 27: 1193-1199.

21. Sari C, Bayram NA, Doğan FE, et al. The evaluation of endothelial functions in patients with coeliac disease. Echocardiography 2012; 29: 471-477.

22. Brar P, Kwon GY, Holleran S, et al. Change in lipid profile in coeliac disease: beneficial effect of glutenfree diet. Am J Med 2006; 119: 786-790.

23. Taavela J, Kurppa K, Collin P, et al. Degree of damage to the small bowel and serum antibody titers correlate with clinical presentation of patients with coeliac disease. Clin Gastroenterol Hepatol 2013; 11: 166-171. 
24. Zanini B, Mazzoncini E, Lanzarotto F, et al. Impact of gluten-free diet on cardiovascular risk factors. A retrospective analysis in a large cohort of coeliac patients. Dig Liver Dis 2013; 45: 810-815.

25. Hallert C, Grant C, Grehn S, et al. Evidence of poor vitamin status in coeliac patients on a gluten-free diet for 10 years. Aliment Pharmacol Ther 2002; 16: 1333-1339.

26. Lewis NR, Sanders DS, Logan RF, Fleming KM, Hubbard RB, West J. Cholesterol profile in people with newly diagnosed coeliac disease: a comparison with the general population and changes following treatment. Br J Nutr 2009; 102: 509-513.
27. Martin J, Geisel T, Maresch C, Krieger K, Stein J. Inadequate nutrient intake in patients with coeliac disease: results from a German dietary survey. Digestion 2013; 87: 240-246.

28. Bhattacharya M, Lomash A, Sakhuja P, Dubey AP, Kapoor S. Clinical and histopathological correlation of duodenal biopsy with IgA anti-tissue transglutaminase titers in children with coeliac disease. Indian J Gastroenterol 2014; 33: 350-354. 